\title{
Immunostaining of Increased Expression of Enhancer of Zeste Homolog 2 (EZH2) in Diffuse Midline Glioma H3K27M-Mutant Patients with Poor Survival
}

\author{
Vega Karlowee ${ }^{\mathrm{a}, \mathrm{d}}$ Vishwa Jeet Amatya ${ }^{\mathrm{b}}$ Takeshi Takayasu $^{\mathrm{a}}$ Motoki Takano ${ }^{\mathrm{a}}$ \\ Ushio Yonezawa $^{a}$ Yukio Takeshima $^{b}$ Kazuhiko Sugiyamac ${ }^{c}$ Kaoru Kurisu $^{a}$ \\ Fumiyuki Yamasaki $^{a}$ \\ a Department of Neurosurgery, Graduate School of Biomedical and Health Sciences, Hiroshima University, \\ Hiroshima, Japan; bepartment of Pathology, Graduate School of Biomedical and Health Sciences, Hiroshima \\ University, Hiroshima, Japan; ' Department of Clinical Oncology \& Neuro-Oncology Program, Hiroshima University, \\ Hiroshima, Japan; ${ }^{d}$ Department of Anatomical Pathology, Diponegoro University, Semarang, Indonesia
}

\section{Keywords}

Diffuse midline glioma · H3K27M mutant · EZH2 .

Immunohistochemical staining

\begin{abstract}
Introduction: The interaction of $\mathrm{K} 27 \mathrm{M}$ mutation in histone $\mathrm{H} 3$ (H3K27M mutation) with polycomb repressive complex 2 (PRC2) is facilitated by the enhancer of zeste homolog 2 $(\mathrm{EZH} 2)$. Subsequently, this interaction leads to the global reduction level of $\mathrm{H} 3 \mathrm{~K} 27$ me3. We analyzed the EZH2 expression level in H3K27M mutation-positive tumors and revealed the association of high EZH2 expression with poor survival. Methods: Our study included 12 patients, with an age range of 6-56 years and treated between 2007 and 2016. All patients underwent MRI study for nonenhanced T1, T2, diffusion, gadolinium-enhanced T1-weighted imaging, and fluid-attenuated inversion recovery (FLAIR). Immunohistochemical staining was performed against H3K27M, H3K27me3, EZH2, EED, mutant isocitrate dehydrogenase 1 (IDH1), a-thalassemia X-linked intellectual disability (ATRX), p53, O6-methylguanine-DNA methyltransferase (MGMT), and Ki-67 antibodies. Results: All
\end{abstract}

patients were negative for IDH1R132H and H3K27me3, but H3K27M-positive. Staining against EZH2 was negative in all histological features of grade II cases (3/12) and positive in grade III and IV cases; EZH2 positivity is associated with poor prognosis $(p=0.0082)$. EZH2 positivity was not associated with EED positivity. Retained ATRX staining was found mostly in grade III and IV cases (6/12). P53 was predominantly positive in cases of astrocytoma and glioblastoma (8/12). The labeling index of $\mathrm{Ki}-67$ was $1.2-31.4 \%$ for grade II and III histological features and $11.2-24.8 \%$ for grade IV. Conclusion: We suggest that the expression of EZH2 is not associated with the PRC2 pathway and increases in patients with H3K27M-mutant diffuse midline glioma and a poor prognosis. Further studies are necessary to understand the mechanism involved.

(c) 2019 S. Karger AG, Basel

\section{Introduction}

Histone $\mathrm{H} 3$ is 1 of the 5 main histone proteins in the structure of chromatin in eukaryotic cells [1]. Modification of histone influences transcription and other pro-

\section{KARGER}

(c) 2019 S. Karger AG, Basel 
cesses in DNA, and one of these modifications is methylation [2]. Lys 27 histone $\mathrm{H} 3$ is methylated by polycomb repressive complex 2 (PRC2), an epigenetic regulator, through its functional enzymatic component, enhancer of zeste homolog 2 (EZH2). In this process, Lys 27 histone $\mathrm{H} 3$ is trimethylated into the stable mark of $\mathrm{H} 3 \mathrm{~K} 27 \mathrm{me} 3$. $\mathrm{H} 3 \mathrm{~K} 27 \mathrm{me} 3$ plays an important role in epigenetic gene silencing $[3,4]$.

The K27M mutation in histone $\mathrm{H} 3$ (i.e., the H3K27M mutation) is developed from methionine substitution at lysine 27 in histone $\mathrm{H} 3 \mathrm{~F} 3 \mathrm{~A}$ and the HIST1H3B/C gene [5, 6]. H3K27M also interacts with PRC2-EZH2 and causes inhibition of PRC2 function, disrupting the H3K27me3 and PRC2-EZH2 interaction. As a result, this mutation leads to a global reduction in $\mathrm{H} 3 \mathrm{~K} 27 \mathrm{me} 3$ [7], which may further result in a loss of function of epigenetic gene silencing. Many studies have shown that EZH2 activation is involved in many kinds of cancer development and progression; EZH2 is therefore considered to be a therapeutic target in certain cancers [8-12].

Diffuse midline glioma is an infiltrative glioma located in the brain stem, thalamus, and spinal cord, with predominantly astrocytic differentiation. Sequencing studies of these gliomas have shown recurrent mutations in the histone H3.3. This tumor is defined as grade IV in the WHO 2016 update because of the poor prognosis [13]. Subsequently, pertaining to the detection of $\mathrm{H} 3 \mathrm{~K} 27 \mathrm{M}$ mutation protein expression, immunohistochemical staining has been confirmed to have a reliable sensitivity and specificity in glioma even when compared to the whole-exome sequencing result $[14,15]$. However, the role of EZH2 in diffuse midline glioma has not been determined yet.

In spite of the WHO 2016 update grading, some recent studies found that diffuse midline glioma H3K27M has more variety in its histomorphology, i.e., features of WHO grades I-IV, and that the prognosis of the patients is not always poor [16-19]. In this study, we focused on the H3K27M mutation immunopositive tumors. We analyzed the EZH2 expression level and revealed its association with the poor survival of patients with H3K27M mutant-positive tumors.

\section{Patient and Methods}

Patients were treated in our hospital between 2007 and 2016. Of the 12 patients, 8 were males and 4 were female, with age range of 6-56 years. Based on the histomorphological features, there were 6 cases of glioblastoma, 2 anaplastic astrocytomas, 1 anaplastic oligodendrogliomas, 2 diffuse astrocytomas, and 1 oligodendroglioma.

Increased EZH2 and Poor Survival in H3K27M-Mutant Glioma Patients
All patients underwent MRI study for nonenhanced T1weighted imaging, T2-weighted imaging, fluid-attenuated inversion recovery (FLAIR), diffusion-weighted imaging (DWI), and gadolinium-enhanced T1-weighted imaging. Cystic components were differentiated as hyper- and hypointense areas on T2-weighted MRI and FLAIR images, respectively. Necrotic components were differentiated on contrast-enhanced T1-weighted images as the interior nonenhancing parts of enhanced lesions. Hemorrhagic lesions were differentiated on nonenhanced T1-weighted MR images as areas of hyperintensity.

Tissue samples were obtained from surgical procedures. All samples were fixed with $10 \%$ buffer formalin, processed as formalin-fixed paraffin-embedded (FFPE) tissue, and stained with hematoxylin-eosin for routine histopathological diagnosis.

Statistical analyses were done with PRISM v7.0 (GraphPad Software Inc, La Jolla, CA, USA). The survival time of H3 K27Mmutant immunopositive patients was measured from the time of diagnosis by MRI to the time of death or last follow-up (range 3.7-80.9 months; median 14.67 months). Factors analyzed as the potential prognostic markers included: hemorrhagic characteristics at diagnosis, gadolinium enhancement, DWI high intensity, or p53, ATRX, and EZH2 immunopositivity. Kaplan-Meier's survival analysis (log-rank test), incorporating each prognostic factor, was performed to evaluate prognostic value in H3K27M-mutant immunopositive patients. For all statistical results, significance was assigned when the $p$ value was $<0.05$.

\section{Immunohistochemical Staining}

Immunostaining was performed with automated immunostainers (BenchMark GX; Ventana, Tucson, AZ, USA) using ultraView Universal DAB detection kit (cat. No. 760-500, Ventana Medical Systems, Tucson, AZ, USA). Slides were deparaffinized and treated with heat-induced epitope retrieval (HIER) using cell conditioning (CC)-1 (cat. No. 950-124, Ventana Medical Systems). The antibodies were rabbit polyclonal anti-H3K27M (\#ABE419, Millipore, Billercia, MA, USA; $1 \mu \mathrm{g} / \mathrm{mL}$ ); rabbit monoclonal anti-H3K27me3 (\#9733, CST, Danvers, MA, USA; $13.33 \mu \mathrm{g} /$ $\mathrm{mL})$; rabbit monoclonal anti-EZH2 (790-4651, Ventana Medical Systems; $15.9 \mu \mathrm{g} / \mathrm{mL}$ ); mousemonoclonalanti-humanIDH1R132H (DIA-H09, Dianova, Hamburg, Germany; $20 \mu \mathrm{g} / \mathrm{mL}$ ); rabbit polyclonal anti-ATRX (HPA001906, Sigma-Aldrich, St. Louis, MO, USA; $5 \mu \mathrm{g} / \mathrm{mL}$ ); monoclonal mouse anti-human p53 protein (NCL-L-p53-DO7, Leica, Newcastle, UK; $10 \mu \mathrm{g} / \mathrm{mL}$ ); monoclonal mouse anti-human Ki-67 antigen clone MIB1 (F7268, Dako, Denmark; $40 \mu \mathrm{g} / \mathrm{mL}$ ); monoclonal mouse anti-EED antibody (163C, Abcam, Cambridge; $10 \mu \mathrm{g} / \mathrm{mL}$ ) and monoclonal rabbit anti-MGMT (EPR4397, Abcam; $5 \mu \mathrm{g} / \mathrm{mL}$ )

$\mathrm{H} 3 \mathrm{~K} 27 \mathrm{M}$ and $\mathrm{H} 3 \mathrm{~K} 27 \mathrm{me} 3 \mathrm{immunostaining} \mathrm{was} \mathrm{performed} \mathrm{us-}$ ing the ultraView template protocol. Slides were treated with HIER for $30 \mathrm{~min}$ and incubated with antibody for $32 \mathrm{~min}$ at $37^{\circ} \mathrm{C}$. $\mathrm{EZH} 2$ staining was performed according to the manufacturer's instructions. Immunostaining for IDH1R132H and ATRX used the ultraView template with HIER for $60 \mathrm{~min}$, and antibody incubation was for 40 and $32 \mathrm{~min}$, respectively. Staining for p53 and Ki-67 (MIB1) used the following customized protocol: HIER treatment for $60 \mathrm{~min}$, peroxidase blocking reagent (S2023, Dako) application for $10 \mathrm{~min}$, and antibody incubation for $40 \mathrm{~min}$. Secondary antibody (414151F, Nichirei Bioscience Inc., Tokyo, Japan) incubation was for $40 \mathrm{~min}$, and was visualized with $3,3^{\prime}$-diaminobenzidine tetrahydrochloride (DAB) (425312F, Nichirei Bioscience Inc.) and 
Table 1. Imaging characteristics of $\mathrm{H} 3 \mathrm{~K} 27 \mathrm{M}$ immunopositive tumors

\begin{tabular}{|c|c|c|c|c|c|c|c|c|c|c|c|c|c|c|}
\hline $\begin{array}{l}\text { Case } \\
\text { No. }\end{array}$ & $\begin{array}{l}\text { Age, } \\
\text { years }\end{array}$ & Sex & $\begin{array}{l}\text { Main tumor } \\
\text { location }\end{array}$ & $\begin{array}{l}\text { T2/ } \\
\text { FLAIR }\end{array}$ & DWI & $\begin{array}{l}\text { Gadolinium } \\
\text { enhancement }\end{array}$ & ITH & $\begin{array}{l}\text { Cyst } \\
\text { formation }\end{array}$ & $\begin{array}{l}\text { Operation } \\
\text { (removal) }\end{array}$ & $\begin{array}{l}\text { Local } \\
\text { RT dose } \\
\text { (Gray) }\end{array}$ & Chemotherapy & $\begin{array}{l}\text { Dissemination + } \\
\text { distant } \\
\text { recurrence }\end{array}$ & OS & $\begin{array}{l}\text { Dead/ } \\
\text { alive }\end{array}$ \\
\hline 2 & 48 & M & Thalamus & High & High & Partly & - & - & Biopsy & 54 & TMZ & + & 23.70 & Dead \\
\hline 3 & 29 & $\mathrm{~F}$ & Thalamus & High & High & Partly & - & - & Partial & 60 & TMZ/BEV & + & 13.43 & Dead \\
\hline 4 & 31 & M & Thalamus & High & High & Homogeneously & - & - & Biopsy & 60 & $\mathrm{TMZ}$ & + & 14.67 & Dead \\
\hline 7 & 6 & $\mathrm{~F}$ & Thalamus & High & High & Heterogenously & + & - & Biopsy & 60 & $\mathrm{BEV}$ & + & 14.87 & Dead \\
\hline 8 & 14 & $\mathrm{~F}$ & Thalamus & High & High & Partly & - & + & Biopsy & 60 & ACNU/CDDP & + & 14.53 & Dead \\
\hline 9 & 21 & M & Thalamus & High & High & Heterogenously & + & + & Biopsy & 60 & TMZ & + & 11.03 & Alive \\
\hline 10 & 30 & M & Pons & High & Iso & None & - & - & Biopsy & 54 & TMZ & + & 28.37 & Dead \\
\hline 11 & 11 & M & Pons-medulla & High & Low & Partly & - & - & Biopsy & 54 & TMZ/BEV & + & 19.40 & Dead \\
\hline 12 & 16 & M & Pons-medulla & High & High & Partly & - & - & Biopsy & 54 & TMZ/BEV & - & 10.40 & Dead \\
\hline
\end{tabular}

F, female; M, male; DWI, diffusion-weighted imaging; ITH, intratumoral hemorrhage; RT, radiation therapy; OS, overall survival; TMZ, temozolomide; $\mathrm{BEV}$, bevacizumab; ACNU, nimustine; CDDP, cisplatin.

copper solution for 8 min each. All counterstains were done manually with hematoxylin for $30 \mathrm{~s}$ and blueing reagent for $5 \mathrm{~s}$.

Evaluation of immunostaining was done at 3 different sites, based on the previous criteria. H3K27M immunopositivity was defined by $>80 \%$ of tumor cells with strong nuclear staining [14], and there was at least $71 \%$ positivity for $\mathrm{H} 3 \mathrm{~K} 27 \mathrm{me} 3$ in the cell nuclei [15]. Positivity for EZH2 staining was determined based on strong nuclei staining [20]. Immunostaining was positive for IDH1R132H with strong cytoplasmic staining [21]. The ATRX and p53 staining positive cut-off value was $10 \%$ nuclear staining [22, 23]. Labeling index evaluation for Ki-67 was based on nuclear staining in tumor cells [24].

\section{Results}

MRI features are summarized in Table 1. Most of the 12 patients were adults, with a tumor located in the thalamus $(n=9)$ and features of glioblastoma WHO grade IV $(n=6)$. All tumors showed high intensity on T2/FLAIR images. Gadolinium enhancement was observed in 83\% (10/12 patients) and showed high intensity on DWI (10/12 patients). Intratumoral hemorrhage is the most important characteristic of $\mathrm{H} 3 \mathrm{~K} 27 \mathrm{M}$ mutant-positive thalamic glioma and was observed in $44.4 \%$ (4/9 patients) of the thalamic tumors, while no brain stem tumors showed hemorrhagic characteristics. Dissemination and distant/remote recurrence are characteristics of H3K27M mutant-positive tumors and were observed in 75\% (9/12) of our series of patients. Prognosis was poor and median overall survival was 14.7 months. Median overall survival of patients with thalamic and brain stem tumors was 14.7 and 19.4 months, respectively $(p>0.05)$.

Immunohistochemical staining is summarized in Table 2. All patients with $\mathrm{H} 3 \mathrm{~K} 27 \mathrm{M}$-positive staining revealed IDH1R132H and H3K27me3 negativity. Staining against EZH2 was negative in all histological features of grade II cases (3/12) and positive in grade III and IV cases. EED was positive in 8/11 tumors and was not associated with WHO grade or EZH2 positivity. MGMT expression was positive in 6/12 patients. Retained ATRX staining was found mostly in grade III and IV cases $(6 / 12)$ and in 1 grade II case. P53 staining was predominantly positive in the cases of astrocytoma and glioblastoma (8/12). The labeling index of Ki-67 was $1.2-31.4 \%$ for grade II and III histological features and $11.2-24.8 \%$ for grade IV glioblastoma. Representative cases of our series are shown in Figures 1-3.

We compared the prognosis of patients with $\mathrm{H} 3 \mathrm{~K} 27 \mathrm{M}$ mutant-positive tumors by taking into account the clinical factors. Between brain stem- and thalamus-located tumor groups, there was no statistical difference (Fig. 4a; $p>0.05)$. There is a poorer prognosis for patients with hemorrhagic tumors than for those with nonhemorrhagic tumors. However, our result did not show any statistical difference (Fig. $4 \mathrm{~b} ; p=0.1471$ ).

Based on the immunohistochemical staining, we found a significant difference between patients with high and low EZH2 expression (Fig. 4c; $p=0.0082$ ). The median overall survival of these 2 groups was 14.5 and 28.4 months, respectively. Furthermore, the overall survival 
Table 2. Immunohistochemical characteristics of $\mathrm{H} 3 \mathrm{~K} 27 \mathrm{M}$ immunopositive tumors

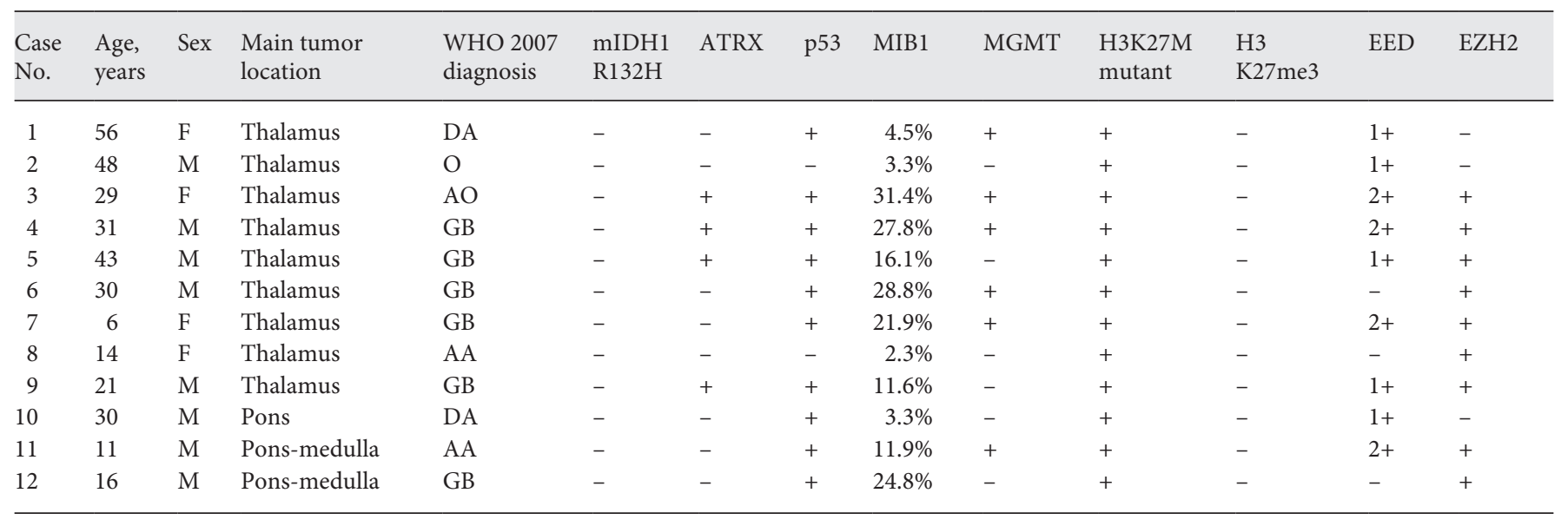

DA, diffuse astrocytoma; O, oligodendroglioma; AA, anaplastic astrocytoma; AO, anaplastic oligodendroglioma; GB, glioblastoma; F, female; M, male; -, negative; +, positive; $1+$, partial positive; $2+$, strong positive.

time of the patients with EZH2-positive thalamic glioma was significantly shorter than that of EZH2-negative thalamic glioma patients ( $p=0.0355 ; 14.5$ vs. 52.3 months; data not shown).

We also analyzed the relationship between tumor grade and staining of EZH2, EED, ATRX, and p53 expression. We found that only EZH2 was statistically associated with tumor grade, and high-grade tumors showed positive expression of EZH2 $(p=0.0045$, Fisher's exact test). No other staining was associated with grade $(p>$ 0.05). Compared to EZH2-negative tumors, the positive ones showed a tendency of a high MIB1 labeling index, but considering the small sample size, the result did not show statistical difference $(p=0.0591)$.

Regarding prognosis, EED expression status was not associated with prognosis ( $p>0.05$; data not shown). ATRX-positive and p53-positive staining as well as MGMT expression status, were not associated with prognosis ( $p>0.05$; data not shown). In addition, we could not find any relationship between immunohistochemical staining results and clinical or imaging characteristics, including surgical achievement, irradiation dose, chemotherapy, dissemination/distant recurrence, hemorrhage, enhancement, and DWI intensity.

\section{Discussion}

Histone modification can be associated with chromatin function and gene activity change in cancer [25]. Lysine 27 trimethylation on histone $\mathrm{H} 3$ (H3K27me3) func- tion is important in lineage specification through developmental gene silencing $[25,26]$. In the homeotic gene expression function, PRC2 methylates lysine 27 on histone $\mathrm{H} 3$ as a repressive chromatin [27]. This methyltransferase activity is mediated by multiple interactions of the PRC2 components. H3K27me3 acts as a stable marker bound to PRC2 as well as activating the PRC2 complex [27]. The cellular H3K27me3 level is determined by the PRC2-EZH2 site and a positive feedback interaction is maintained $[3,4]$. Consequently, modification on H3K$27 \mathrm{me} 3$ may result in aberrant gene expression and genomic instability [25].

In glioma, H3K27M mutation is associated with a global reduction in H3K27me3 level [7, 15, 20, 28]. Some studies found that the level of the $\mathrm{H} 3 \mathrm{~K} 27 \mathrm{me} 3$ gene promoter is low or even lost in H3K27M glioma [15, 28, 29]. However, the mechanism of this effect remains unclear as the mutation is only present in some of the histones [27]. Mutation of $\mathrm{H} 3 \mathrm{~K} 27 \mathrm{M}$ occurs in relation to mutation in histone $\mathrm{H} 3$ genes (H3F3A and HIST1H3B) resulting in lysine 27 substitution to methionine $[5,6,30]$. H3K27M mutation may influence tumorigenesis through the gene expression reprogramming linked to its interaction with epigenetic regulator PRC2 in the SET domain [27, 28].

PRC2 is susceptible to either gain- or loss-of-function alterations in malignancy [23]. According to the PRC2 structural basis described in Justin et al. [27] (the structural basis of the oncogenic H3K27M inhibition of PRC2), the binding of PRC2 and the nucleosome involves multiple interactions of PRC2 components. These components are SUZ12 and RbAp48 binding to H3, the SET 


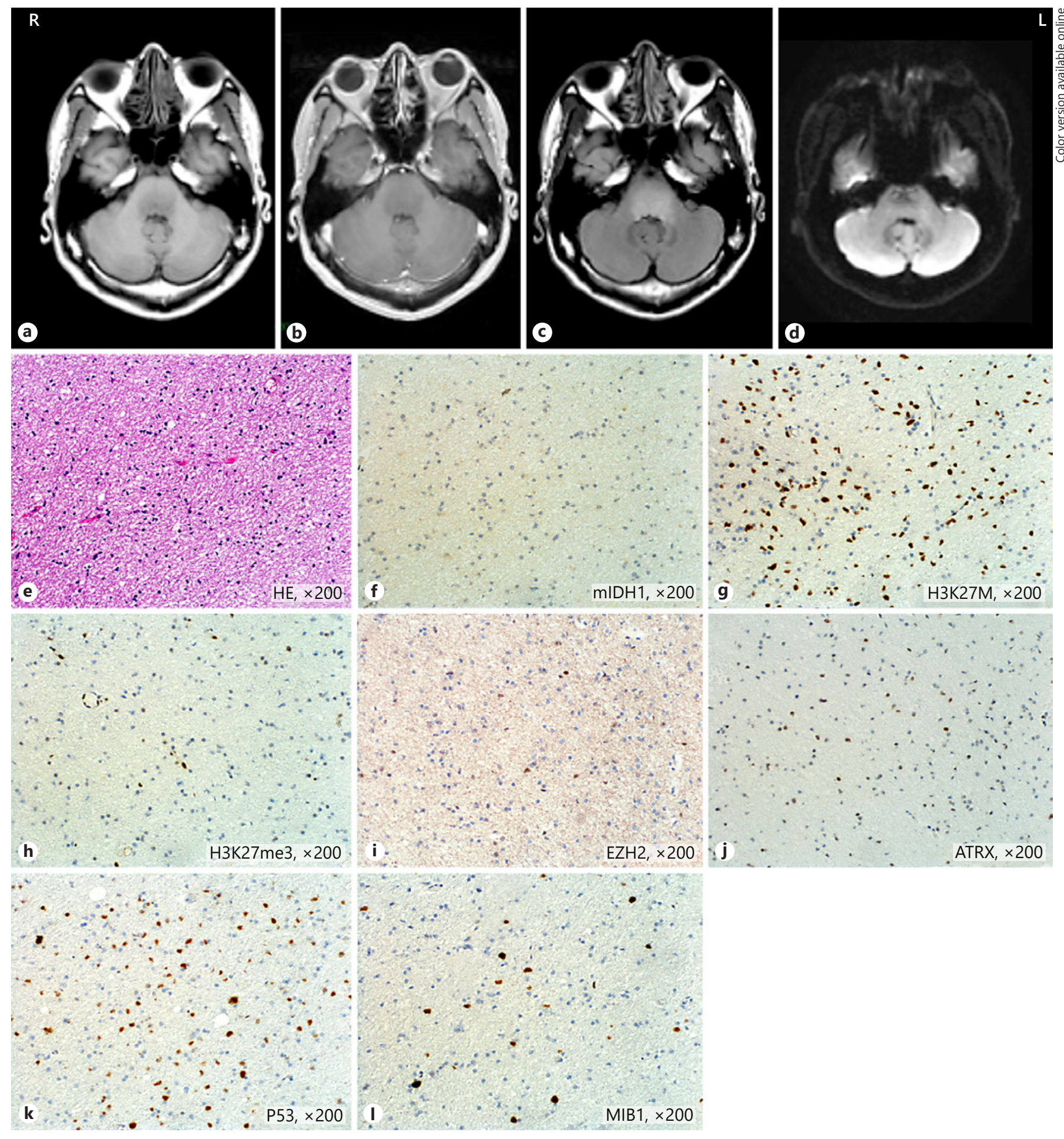

Fig. 1. Radiological and histological features of brain stem diffuse astrocytoma in a 30-year-old male. a Axial T1-weighted image. b Axial T1-weighted image after gadolinium infusion. c FLAIR image (pre-enhancement). d Diffusion-weighted image (pre-enhancement). e Biopsy of the mass revealed a predominantly low

cellularity astrocytic tumor. Mutant IDH1R132 staining was negative (f) and mutant $\mathrm{H} 3 \mathrm{~K} 27 \mathrm{M}$ staining was positive $(\mathbf{g})$. Staining was negative for H3K27me3 (h), EZH2 (i), and ATRX (j) antibodies. k P53 was positive. I Ki-67 labeling index was 3.3\%. 

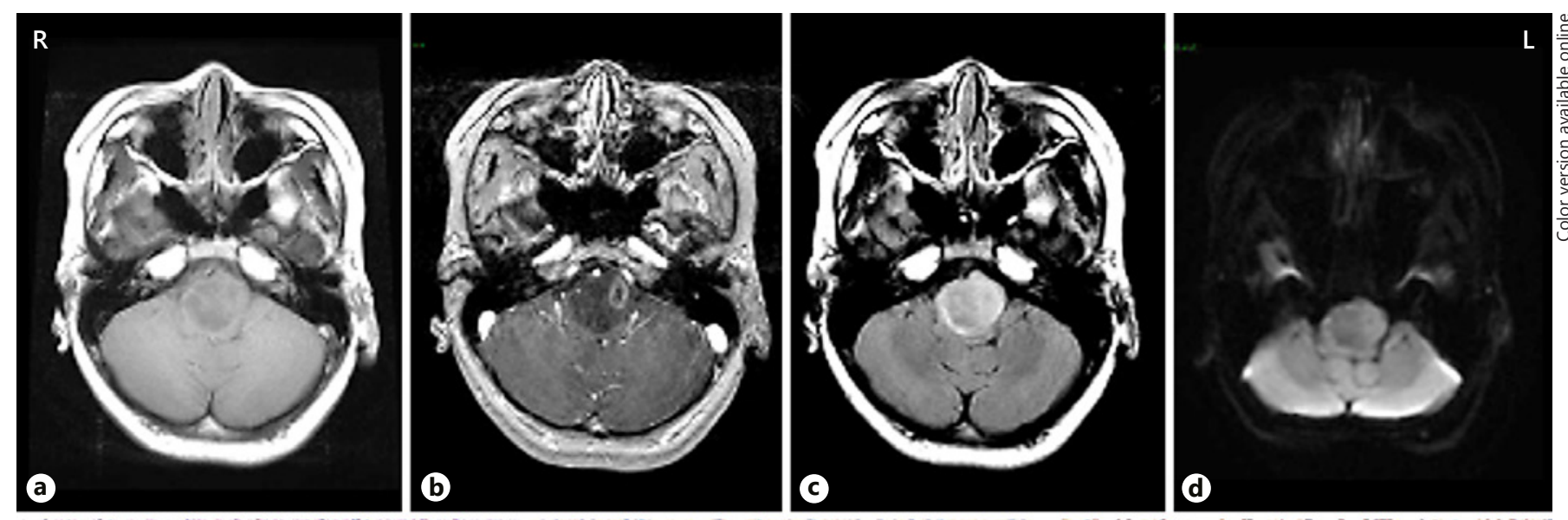

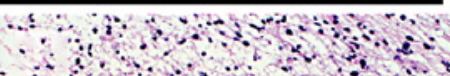

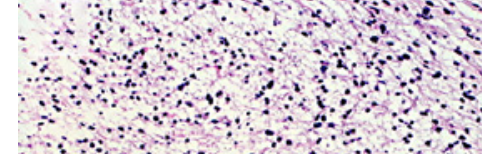

25
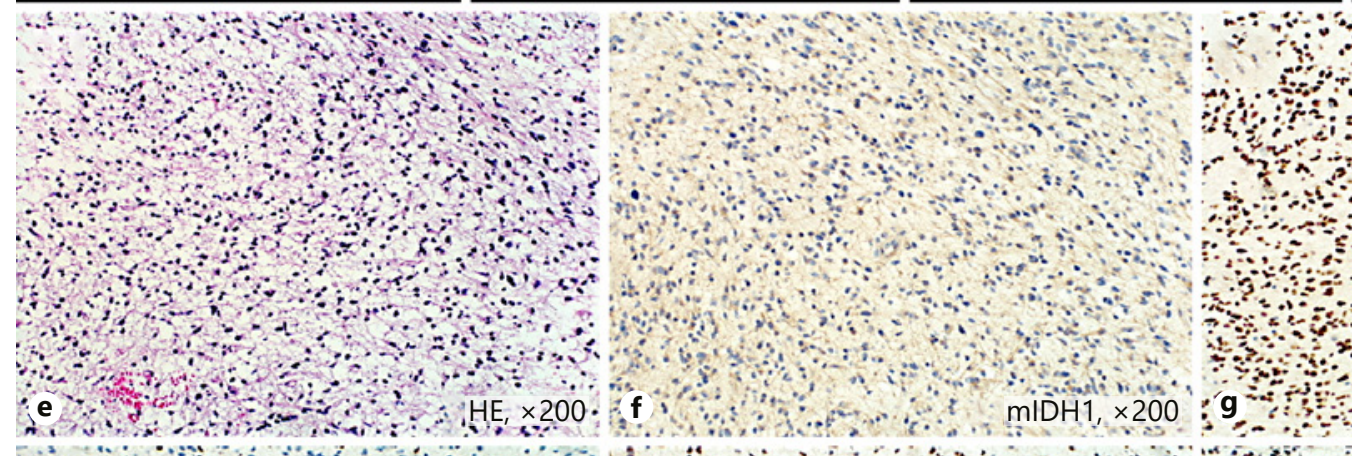

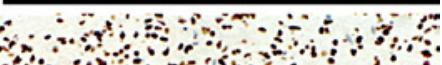
is
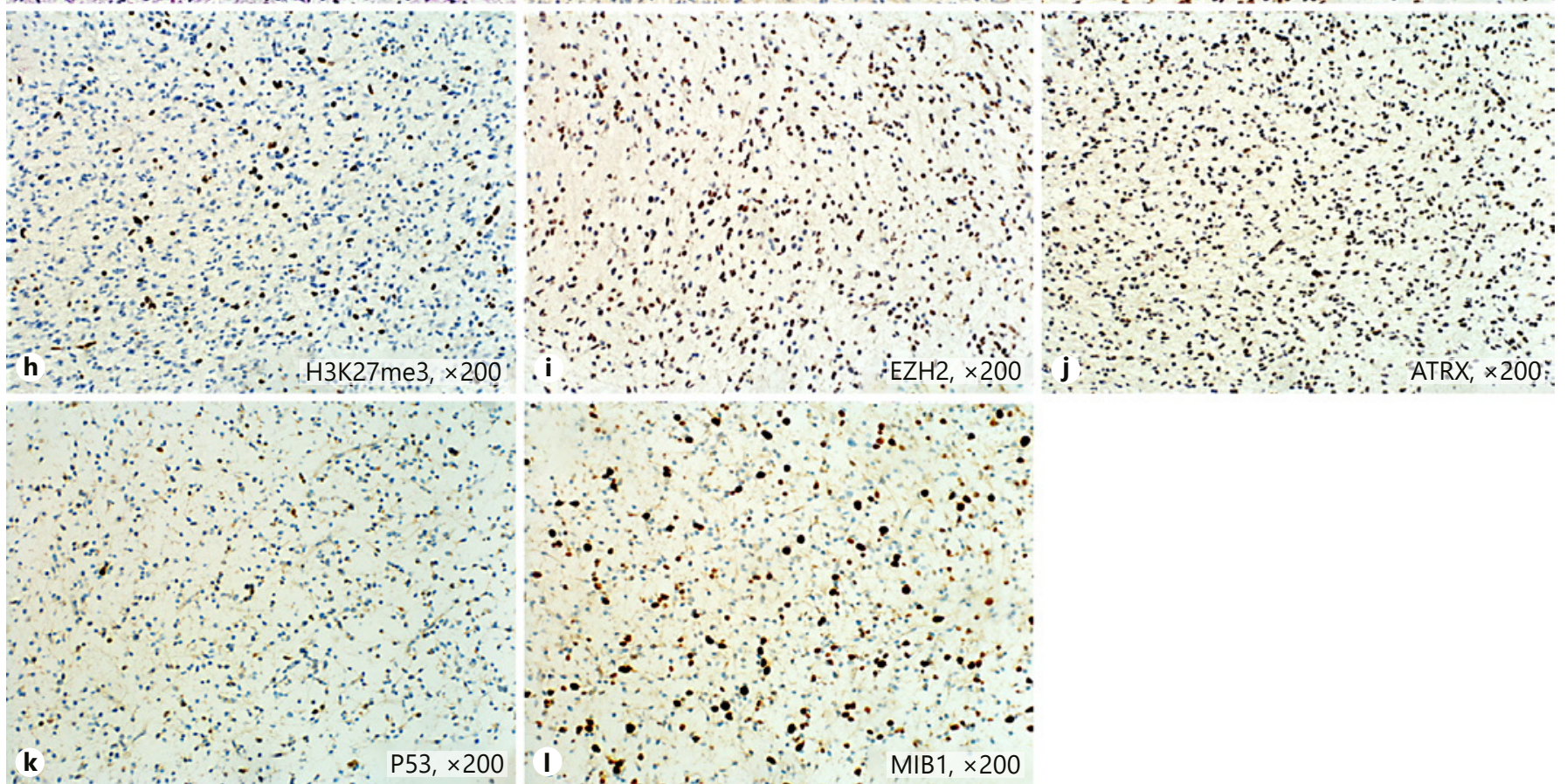

Fig. 2. Radiological and histological features of brain stem anaplastic astrocytoma in an 11-year-old male. a Axial T1-weighted image. b Axial T1-weighted image after gadolinium infusion. c FLAIR image (pre-enhancement). d Diffusion-weighted image (pre-enhancement). e The tissue sample indicated a tumor composed of moderate-to-high cellularity with atypical mitoses. $\mathbf{f}$ The tumor was mutant IDH1R132-negative. Staining for mutant H3K27M was positive (g) while $\mathrm{H} 3 \mathrm{~K} 27 \mathrm{me} 3$ staining was negative (h). EZH2 staining was positive (i), as was ATRX staining (j). P53 was negative (k) with a Ki-67 labeling index of $11.9 \%(\mathbf{I})$. 

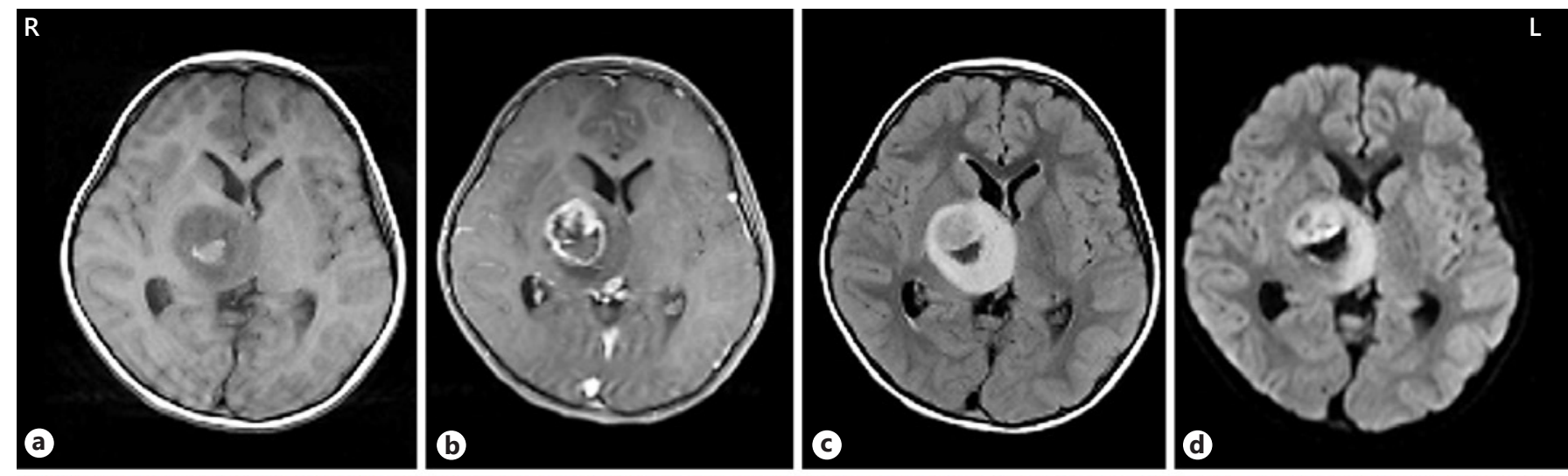

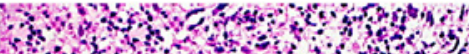
4. 3.

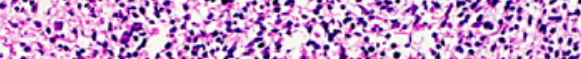

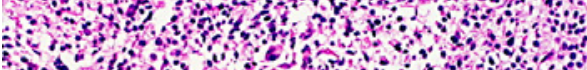

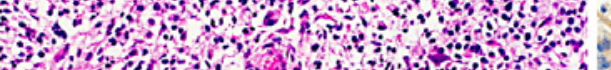
3.0.

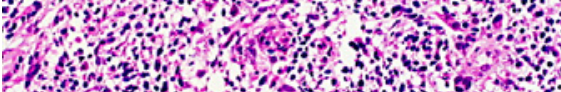

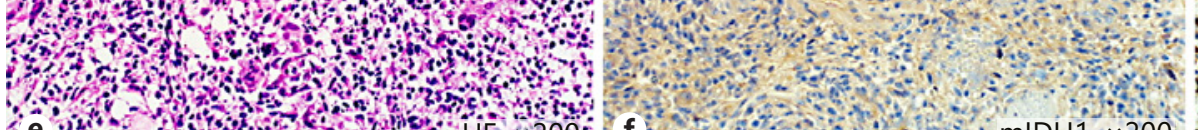

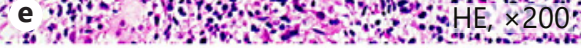

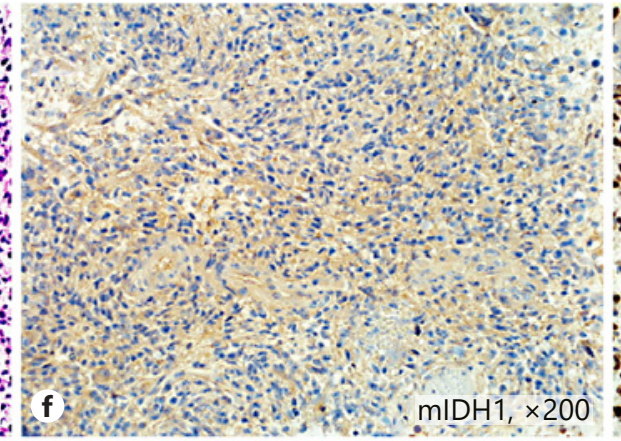

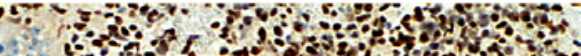

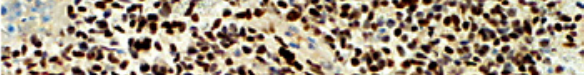
\%

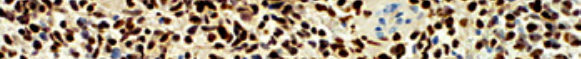

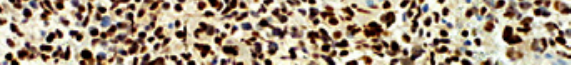

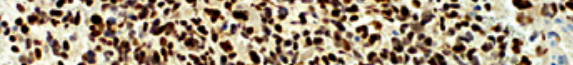

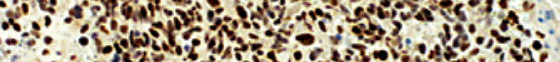
$3 \times$ sing s.t.

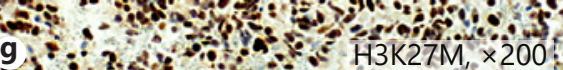

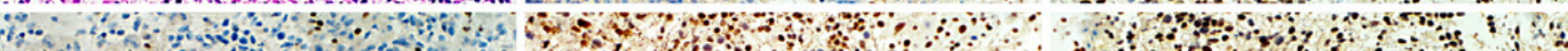
40 a a on 0 a

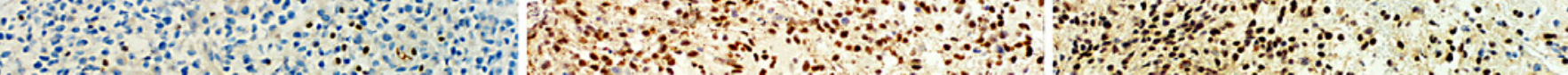

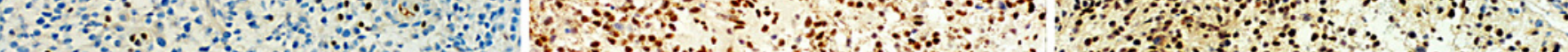

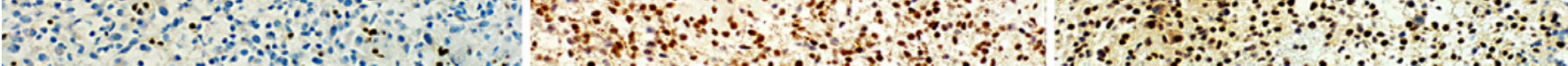

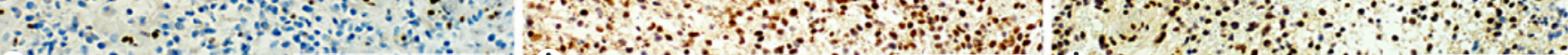

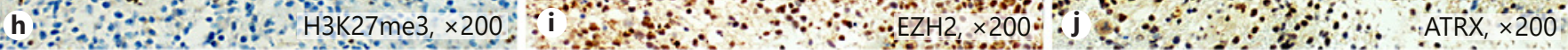

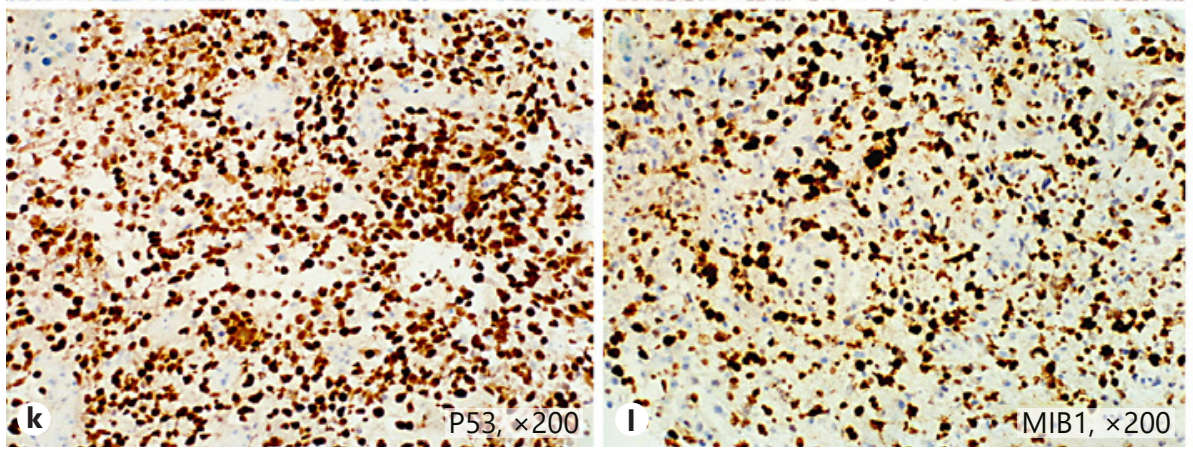

Fig. 3. Radiological and histological features of a thalamic glioblastoma in a 6-year-old female. a Axial T1-weighted image. b Axial T1-weighted image after gadolinium infusion. c FLAIR image (pre-enhancement). d Diffusion-weighted image (pre-enhancement). e Tissue biopsy showed anaplastic tumor nuclei with fre- quent mitoses and perivascular proliferation. $\mathbf{f}$ Mutant IDH1R132 staining was negative. Staining against mutant H3K27M antibody was positive (g) while $\mathrm{H} 3 \mathrm{~K} 27 \mathrm{me} 3$ staining was negative (h). EZH2 (i), ATRX (j), and p53 (k) all showed immunopositivity. I The average Ki-67 labeling index was $21.9 \%$. 


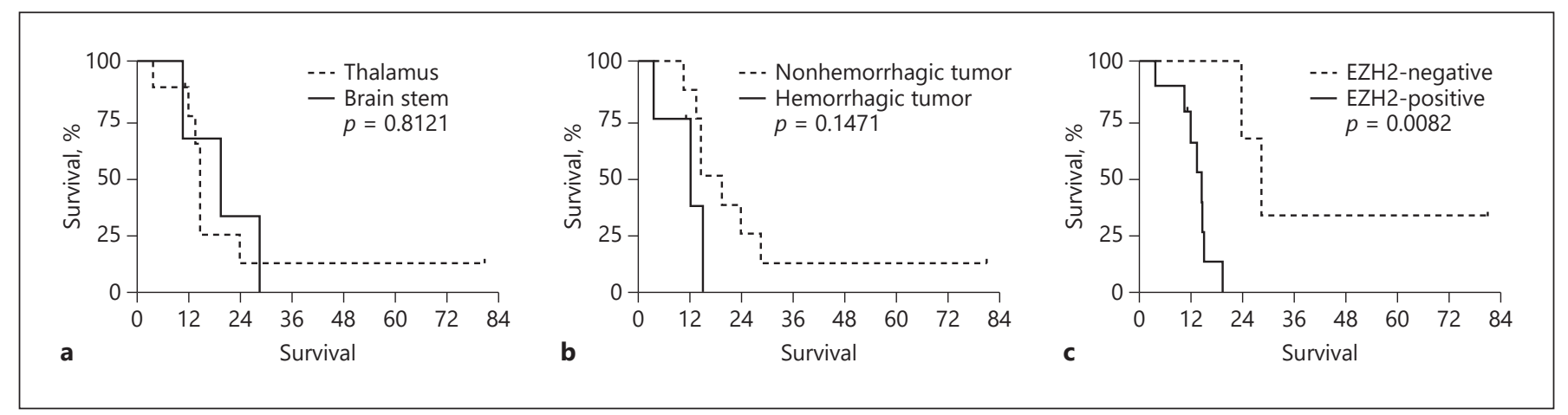

Fig. 4. Kaplan-Meier survival curves illustrating cumulative survival rates for patients of H3 K27M-mutant immunopositive patients. Comparisons were made between thalamic tumors and brain stem tumors (a), hemorrhagic and nonhemorrhagic tumors (b), and EZH2 immunonegative and immunopositive tumors (c).

domain binding to $\mathrm{H} 3 \mathrm{~K} 27$, and $\mathrm{EED}$ binding to $\mathrm{H} 3 \mathrm{~K}$ 27me3. The EZH2 SET domain requires the other PRC2 components for activation and shows autoinhibition without them [27]. Referring to its natural role in gene silencing/repression, PRC2 maintains the determined transcriptional program to preserve cell identity and differentiation $[3,4,31]$. Accordingly, its role in cancer is also dependent on the context of the specific combinations of the tumorigenic alteration, including the regulated genes, the cell of origin, and the developmental history [31].

As one of the active sites of PRC2, EZH2 expression is increased or lost in malignancy [32, 33]. Overexpression of EZH2 has mostly been related to progressive behavior in solid tumors in breast, endometrial, lung, and prostate cancers [8-11]. Cancer with EZH2 overexpression revealed oncogenic activity via the transcriptional activation of EZH2 independent of PRC2 function; for this reason, EZH2 has become an appealing target in tumor therapy [12]. However, a deficiency of EZH2 has also been identified in malignancies like $\mathrm{T}$ cell acute leukemia and myelodysplastic disorders $[34,35]$. Therefore, it is postulated that the role of EZH2 in malignancy is dependent on its affinity to histone and nonhistone substrates [33]. Increased expression of EZH2 has been found to be related to high-grade glioma and poor prognosis in glioblastoma [20, 36-39]. In diffuse midline glioma, the upregulation of transcription activity is also related to the loss of PRC2 repression function [15, 29]. As indicated by Mohammad et al. [40] and Piunti et al. [41], H3K27M tumor also requires PRC2-EZH2 for tumor growth preservation and proliferation [40-42].

Recent studies on diffuse midline glioma have put forward a new understanding regarding the $\mathrm{H} 3$ mutant in diffuse intrinsic pontine glioma. Piunti et al. [41] revealed that $\mathrm{H} 3 \mathrm{~K} 27 \mathrm{me} 3$ reduction is due to the PRC2 exclusion from chromatin. Their data indicate that H3K27M does not involve EZH2, thus excluding PRC2 binding. Subsequently, H3K27M progresses through H3K27 acetylation and represses PRC2 activity [41, 42]. Mohammad et al. [40] added the evidence that H3K27M is excluded from some loci due to DNA methylation [42]. This methylation results in the incorporation of $\mathrm{H} 3 \mathrm{~K} 27 \mathrm{M}$ at the weak polycomb target, but this is not enough to reduce the overall activity of PRC2. Thus, H3K27me3 may be retained at several loci in H3K27M-mutant tumors.

Consistent with other studies $[15,28,29]$, all of the $\mathrm{H} 3 \mathrm{~K} 27 \mathrm{M}$ immunostaining positive cases in our study were negative for H3K27me3. Furthermore, despite varying age and tumor grades, EZH2 expression was higher in high-grade glioma and in patients with a worse prognosis. Although it is still difficult to determine the connection between $\mathrm{H} 3 \mathrm{~K} 27 \mathrm{M}$ and $\mathrm{EZH} 2$, our result correlates with studies that found that PRC2-EZH2 was a requirement for tumor growth and proliferation. Regarding the interaction between PRC2 components, we also found that this EZH2 positivity is not correlated with EED immunostaining expression. This indicates that EZH2 overexpression is independent of the PRC2 activity. Nonetheless, we could not determine whether this role of EZH2 is unique to diffuse midline glioma or also a feature of other gliomas and cancers in general.

With regard to other molecular characteristics in glioma, we also performed immunohistochemical staining against IDH1R132H, ATRX, p53, MGMT, and MIB1. Mutation of IDH1 is mutually exclusive with H3F3A mutation [30,43] and was found to be negative on immunostaining in all $\mathrm{H} 3 \mathrm{~K} 27 \mathrm{M}$ mutation gliomas [16]. 
Mutant IDH1R132H immunostaining has sensitivity of $83 \%$ and a specificity of $100 \%$ compared to direct sequencing [21]. All of our cases showed up as IDH1R132negative, consistent with results of previous studies. TP53 mutation is found in roughly $50 \%$ of the cases of H3K27M mutation [43] and positive p53 expression in $40 \%$ of the cases $[6,44]$. Less frequent than TP53, ATRX mutation is also found in a smaller percentage of H3K27M mutation cases; this mutation includes frameshift insertions/deletions, gains of a stop codon, and missense SNVs, which lead to a loss of expression on immunostaining $[16,26]$. We also found a consistent result, although with slightly higher percentages of both p53-positive and ATRX-negative staining. This was presumably linked to the limited number and variety of cases. MGMT inactivation is correlated with clinical response to alkylating agents like temozolomide [45]. Half of our cases showed positivity. Furthermore, our labeling index of Ki-67 result was higher in glioblastoma and anaplastic glioma features, compared to the grade II feature. This result is consistent with the glioma profile in general [24].

We acknowledge that our study had limitations. We had only a small number of cases with little variety regarding the location, and we did not make any differentiation between adult and pediatric characteristics. The study was also based on only a few components of PRC2 (EED2 and EZH2) with an immunostaining modality, and no DNA analysis was performed regarding the complex interaction among PRC2 components.

In conclusion, our immunostaining profile against $\mathrm{H} 3 \mathrm{~K} 27 \mathrm{M}$ and H3K27me3 was consistent with previous studies on diffuse midline glioma. All tumors with positive staining against $\mathrm{H} 3 \mathrm{~K} 27 \mathrm{M}$ showed a global reduction of $\mathrm{H} 3 \mathrm{~K} 27 \mathrm{me} 3$ expression. Additionally, EZH2 staining increased expression as a clinical progressive factor is also consistent with the previous studies in glioma and other cancers. Accordingly, this finding is interesting with reference to the variety of prognoses despite the tumor grade of WHO IV as well as EZH2 inhibition as a potential target for therapy in diffuse midline glioma.

\section{Acknowledgement}

This study was partially supported by the Japan Society for the promotion of Science Grants-in-Aid for Scientific Research (No. 16K10757).

\section{Disclosure Statement}

All authors have no conflicts of interest.

\section{References}

1 Cutter AR, Hayes JJ. A brief review of nucleosome structure. FEBS Lett. 2015 Oct;589(20 Pt A):2914-22.

2 Bannister AJ, Kouzarides T. Regulation of chromatin by histone modifications. Cell Res. 2011 Mar;21(3):381-95.

3 Margueron R, Reinberg D. The polycomb complex PRC2 and its mark in life. Nature. 2011;469:343-9.

4 Simon JA, Kingston RE. Occupying chromatin: polycomb mechanisms for getting to genomic targets, stopping transcriptional traffic and staying put. Mol Cell. 2013;49(5):80824.

5 Wu G, Broniscer A, McEachron TA, Lu C, Paugh BS, Becksfort J, et al; St. Jude Children's Research Hospital-Washington University Pediatric Cancer Genome Project. Somatic histone $\mathrm{H} 3$ alterations in pediatric diffuse intrinsic pontine gliomas and non-brainstem glioblastomas. Nat Genet. 2012 Jan;44(3): 251-3.

6 Wu G, Diaz AK, Paugh BS, Rankin SL, Ju B, Li Y, et al. The genomic landscape of diffuse intrinsic pontine glioma and pediatric nonbrainstem high-grade glioma. Nat Genet. 2014 May;46(5):444-50.
7 Lewis PW, Müller MM, Koletsky MS, Cordero F, Lin S, Banaszynski LA, et al. Inhibition of PRC2 activity by a gain-of-function H3 mutation found in pediatric glioblastoma. Science. 2013;340(6134):857-61.

8 Michalak EM, Visvader JE. Dysregulation of histone methyltransferases in breast cancer opportunities for new targeted therapies? Mol Oncol. 2016;10(10):1497-515.

9 Jia N, Li Q, Tao X, Wang J, Hua K, Feng W. Enhancer of zeste homolog 2 is involved in the proliferation of endometrial carcinoma. Oncol Lett. 2014 Nov;8(5):2049-54.

10 Ngollo M, Lebert A, Dagdemir A, Judes G, Karsli-Ceppioglu S, Daures $M$, et al. The association between histone 3 lysine 27 trimethylation ( $\mathrm{H} 3 \mathrm{~K} 27 \mathrm{me} 3)$ and prostate cancer: relationship with clinicopathological parameters. BMC Cancer. 2014;14: 994.

11 Dimou A, Dincman T, Evanno E, Gemmill R, Roche J, Drabkin H. Epigenetics during EMT in lung cancer: $\mathrm{EZH} 2$ as a potential therapeutic target. Cancer Treat Res Commun. 2017; 12:40-8.

$12 \mathrm{Kim} \mathrm{KH}$, Roberts $\mathrm{CW}$. Targeting EZH2 in cancer. Nat Med. 2016 Feb;22(2):128-34.
13 Louis DN, Ohgaki H, Wiestler O, Cavenee WK, Ellison DW, Figarella-Branger D, et al, editors. WHO Classification of Tumours of the Central Nervous System. Revised 4th ed. Lyon: IARC; 2016.

14 Venneti S, Santi M, Felicella MM, Yarilin D, Phillips JJ, Sullivan LM, et al. A sensitive and specific histopathologic prognostic marker for $\mathrm{K} 27 \mathrm{M}$ mutant pediatric glioblastomas. Acta Neuropathol (Berl) 1;128(5):743-53.

15 Bender S, Tang Y, Lindroth AM, Hovestadt V, Jones DTW, Kool M, et al. Reduced H3K$27 \mathrm{me} 3$ and DNA hypomethylation are major drivers of gene expression in K27M mutant pediatric high-grade gliomas. Cancer Cell. 2013;24(5):660-72.

16 Solomon DA, Wood MD, Tihan T, Bollen AW, Gupta N, Phillips JJJ, et al. Diffuse Midline Gliomas with Histone H3-K27M mutation: a series of 47 cases assessing the spectrum of morphologic variation and associated genetic alterations. Brain Pathol. 2016;26(5): $569-80$

17 Morita S, Nitta M, Muragaki Y, Komori T, Masui K, Maruyama T, et al. Brainstem pilocytic astrocytoma with $\mathrm{H} 3 \mathrm{~K} 27 \mathrm{M}$ mutation: case report. J Neurosurg. 2018;129(3):593-7. 
18 Pagès M, Beccaria K, Boddaert N, Saffroy R, Besnard A, Castel D, et al. Co-occurrence of histone H3 K27M and BRAF V600E mutations in paediatric midline grade I ganglioglioma. Brain Pathol. 2018;28(1):103-11.

19 Joyon N, Tauziède-Espariat A, Alentorn A, Giry M, Castel D, Capelle L, et al. K27M mutation in $\mathrm{H} 3 \mathrm{~F} 3 \mathrm{~A}$ in ganglioglioma grade I with spontaneous malignant transformation extends the histopathological spectrum of the histone $\mathrm{H} 3$ oncogenic pathway. Neuropathol Appl Neurobiol. 2017;43(3):271-6.

20 Venneti S, Garimella MT, Sullivan LM, Martinez D, Huse JT, Heguy A, et al. Evaluation of Histone 3 Lysine 27 Trimethylation (H3K$27 \mathrm{me} 3$ ) and Enhancer of Zest 2 (EZH2) in pediatric glial and glioneuronal tumors shows decreased H3K27me3 in H3F3A K27M mutant glioblastomas. Brain Pathol. 2013;23(5): 558-64.

21 Capper D, Weissert S, Balss J, Habel A, Meyer J, Jäger D, et al. Characterization of $\mathrm{R} 132 \mathrm{H}$ mutation-specific IDH1 antibody binding in brain tumors. Brain Pathol. 2010 Jan;20(1): 245-54.

22 Wiestler B, Capper D, Holland-Letz T, Korshunov A, Deimling A von, Pfister SM, et al. ATRX loss refines the classification of anaplastic gliomas and identifies a subgroup of IDH mutant astrocytic tumors with better prognosis. Acta Neuropathol (Berl). 2013; 126(3):443-51.

23 Takami H, Yoshida A, Fukushima S, Arita H, Matsushita Y, Nakamura T, et al. Revisiting TP53 mutations and immunohistochemistry: a Comparative study in 157 diffuse gliomas. Brain Pathol. 2015 May;25(3):256-65.

24 Skjulsvik AJ, Mørk JN, Torp MO, Torp SH. Ki-67/MIB-1 immunostaining in a cohort of human gliomas. Int J Clin Exp Pathol. 2014 Dec;7(12):8905-10.

25 Füllgrabe J, Kavanagh E, Joseph B. Histone onco-modifications. Oncogene. 2011;30: 3391.

26 Vastenhouw NL, Schier AF. Bivalent histone modifications in early embryogenesis. Nucl Gene Expr. 2012;24(3):374-86.
27 Justin N, Zhang Y, Tarricone C, Martin SR, Chen S, Underwood E, et al. Structural basis of oncogenic histone H3K27M inhibition of human polycomb repressive complex 2 . Nat Commun. 2016;7:11316

28 Chan K-M, Fang D, Gan H, Hashizume R, Yu C, Schroeder M, et al. The histone H3.3K27M mutation in pediatric glioma reprograms H3K27 methylation and gene expression. Genes Dev. 2013;27(9):985-90.

29 Reddington JP, Perricone SM, Nestor CE, Reichmann J, Youngson NA, Suzuki M, et al. Redistribution of H3K27me3 upon DNA hypomethylation results in de-repression of Polycomb target genes. Genome Biol. 2013; 14:R25.

30 Schwartzentruber J, Korshunov A, Liu XY Jones DT, Pfaff E, Jacob K, et al. Driver mutations in histone H3.3 and chromatin remodelling genes in paediatric glioblastoma. Nature. 2012 Jan;482(7384):226-31.

31 Comet I, Riising EM, Leblanc B, Helin K. Maintaining cell identity: PRC2-mediated regulation of transcription and cancer. Nat Rev Cancer. 2016;16:803.

32 Hock H. A complex Polycomb issue: the two faces of EZH2 in cancer. Genes Dev. 2012; 26(8):751-5.

33 Gall Trošelj K, Novak Kujundzic R, Ugarkovic D. Polycomb repressive complex's evolutionary conserved function: the role of EZH2 status and cellular background. Clin Epigenetics. 2016;8:55.

34 Simon C, Chagraoui J, Krosl J, Gendron P, Wilhelm B, Lemieux S. A key role for EZH2 and associated genes in mouse and human adult T-cell acute leukemia. Genes Dev. 2012; 26(7):651-6.

35 Muto T, Sashida G, Oshima M, Wendt GR, Mochizuki-Kashio M, Nagata Y. Concurrent loss of Ezh2 and Tet2 cooperates in the pathogenesis of myelodysplastic disorders. J Exp Med 2013;210(12):2627-39.

36 Crea F, Hurt EM, Farrar WL. Clinical significance of Polycomb gene expression in brain tumors. Mol Cancer. 2010;9:265.
37 Orzan F, Pellegatta S, Poliani PL, Pisati F, Caldera V, Menghi F, et al. Enhancer of Zeste 2 $(\mathrm{EZH} 2)$ is up-regulated in malignant gliomas and in glioma stem-like cells. Neuropathol Appl Neurobiol. 2011;37(4):381-94.

38 Zhang Y, Yu X, Chen L, Zhang Z, Feng S, Zhang Y, et al. EZH2 overexpression is associated with poor prognosis in patients with glioma. Oncotarget. 2016;8(1):565-73.

39 Zhang J, Chen L, Han L, Shi Z, Zhang J, Pu P. $\mathrm{EZH} 2$ is a negative prognostic factor and exhibits pro-oncogenic activity in glioblastoma. Cancer Lett. 2015;356(2):929-36.

40 Mohammad F, Weissmann S, Leblanc B, Pandey DP, Højfeldt JW, Comet I, et al. EZH2 is a potential therapeutic target for H3K27Mmutant pediatric gliomas. Nat Med. $2017 \mathrm{Apr}$; 23(4):483-92.

41 Piunti A, Hashizume R, Morgan MA, Bartom ET, Horbinski CM, Marshall SA, et al. Therapeutic targeting of polycomb and BET bromodomain proteins in diffuse intrinsic pontine gliomas. Nat Med. 2017 Apr;23(4):493500 .

42 Creasy CL. Untangling the role of mutant histone $\mathrm{H} 3$ in diffuse intrinsic pontine glioma. Nat Med. 2017 Apr;23(4):413-4.

43 Khuong-Quang D-A, Buczkowicz P, Rakopoulos P, Liu X-Y, Fontebasso AM, Bouffet E, et al. K27M mutation in histone H3.3 defines clinically and biologically distinct subgroups of pediatric diffuse intrinsic pontine gliomas. Acta Neuropathol (Berl). 2012;124(3):43947.

44 Castel D, Philippe C, Calmon R, Le Dret L, Truffaux N, Boddaert $\mathrm{N}$, et al. Histone H3F3A and HIST1H3B K27M mutations define two subgroups of diffuse intrinsic pontine gliomas with different prognosis and phenotypes. Acta Neuropathol. 2015 Dec; 130(6):815-27.

45 Capper D, Mittelbronn M, Meyermann R, Schittenhelm J. Pitfalls in the assessment of MGMT expression and in its correlation with survival in diffuse astrocytomas: proposal of a feasible immunohistochemical approach. Acta Neuropathol. 2008 Feb;115(2):249-59. 